\title{
OPTIMIZACIÓN DEL PROCESO DE INMOVILIZACIÓN DE LIPASA PRODUCIDA POR Marinobacter sp CH27 EN OCTADECILO-SEPABEAD
}

\author{
Yadira Fernández Jeri ${ }^{* a}$, Amparo I. Zavaleta ${ }^{a}$, Ana María Muñoz Jáuregui ${ }^{b}$
}

\begin{abstract}
RESUMEN
El objetivo de esta investigación fue optimizar el proceso de inmovilización de la lipasa producida por Marinobacter sp $\mathrm{CH} 27$ en el soporte hidrofóbico octadecilo-sepabead por adsorción interfacial utilizando el diseño experimental Box-Behnken a 3 factores y 3 niveles con 12 experimentos y 3 réplicas en el punto central. Las variables y los niveles seleccionados fueron: fuerza iónica $(2,5 ; 5,0$ y 10,0 mM), tiempo $(30,60$ y $120 \mathrm{~min})$ y pH $(6,0 ; 7,0$ y 8,0$)$ y las variables respuestas fueron la actividad de la enzima inmovilizada y el porcentaje de inmovilización. Los parámetros óptimos del proceso de inmovilización fueron: $\mathrm{pH}$ 6,62; 30 min y fuerza iónica $10 \mathrm{mM}$. Luego de ajustar los parámetros al modelo polinomial cuadrático, la actividad de lipasa inmovilizada fue de 11,16 U/g soporte con $81,50 \%$ y $74,0 \%$ de actividad relativa y rendimiento de inmovilización, respectivamente.
\end{abstract}

Palabras clave: Lipasa, Marinobacter sp, inmovilización, octadecilo sepabead, metodología superficie respuesta

\section{OPTIMIZATION OF THE PROCESS OF IMMOBILIZATION OF LIPASE PRODUCED BY Marinobacter sp CH27 IN OCTADECILO - SEPABEAD}

\begin{abstract}
The aim of these research was the optimize in the support hydrophobic octadecilo-sepabead by interfacial adsorption, for which the experimental design was used Box-Behnken 3 factors and 3 levels with a total of 12 experiments and 3 replicas on the central point. The variables and their levels selected were: force Ionic $(2.5,5.0$ and $10.0 \mathrm{mM})$, time $(30,60$ and 120 $\min )$ and $\mathrm{pH}(6.0 ; 7.0$ and 8.0$)$ and them variable answers were the activity of the enzyme immobilized (U/g support) and the percentage immobilization. The optimum parameters of the immobilization process were: $\mathrm{pH} 6.62,30 \mathrm{~min}$ and ionic strength $10 \mathrm{Mm}$. After adjust the parameters to the model polynomial quadratic, is obtained 11,16 U/g support for the
\end{abstract}

\footnotetext{
${ }^{a}$ Facultad de Farmacia y Bioquímica, Universidad Nacional Mayor de San Marcos, Jr. Puno 1002, Lima 1, Perú. *yfernandezj@unmsm.edu.pe

b Facultad de Ciencias de la Salud, Universidad San Ignacio de Loyola.
} 
activity of lipase immobilized, with $81.50 \%$ and $74.0 \%$ of activity relative and performance of immobilization respectively.

Key words: Lipase, Marinobacter sp, inmovilization, octadecyl - sepabead, response surface methodology.

\section{INTRODUCCIÓN}

Las lipasas presentan diversas propiedades catalíticas que sustentan su empleo en las industrias: alimentaria, farmacéutica, textil, cosmética, papelera, peletera y en detergentes. En los últimos años, las lipasas se usan en la síntesis de biopolímeros y biodiesel, en la producción de medicamentos enantioselectivos y agroquímicos ${ }^{1}$. A nivel industrial, los mecanismos catalíticos de las lipasas se asemejan a los procesos metabólicos naturales, siendo biodegradables y limpios. Por las aplicaciones antes descritas, las lipasas producidas por nuevos microorganismos están en constante investigación.

El interés en el desarrollo tecnológico de biocatalizadores se centra en la inmovilización de enzimas, lo cual permite mejorar propiedades térmicas con estabilidad en un rango deseado de $\mathrm{pH}$ y en presencia de solventes orgánicos, que sean de fácil recuperación, permitan obtener un producto no contaminado, e incrementar la estabilidad operacional del biocatalizador ${ }^{2,3}$.

Uno de los protocolos de inmovilización de lipasas es la adsorción selectiva sobre soportes hidrofóbicos, para su uso en solventes acuosos y orgánicos. La adsorción involucra áreas hidrofóbicas alrededor del centro activo, quedando en su forma abierta accesible al sustrato. Así, los soportes hidrofóbicos empleados se comportan como los sustratos naturales de estas enzimas ${ }^{4}$.

El soporte hidrofóbico octadecilo sepabead está conformado de una matriz polimérica de metacrilato cubierto por grupos octadecilos que proporcionan una superficie fuertemente hidrofóbica. Este soporte presenta estabilidad química y térmica, resistencia al cambio osmótico, alta capacidad de unión a proteínas, baja tendencia al hinchamiento en soluciones de elevada molaridad y en solventes ${ }^{5}$.

Marinobacter sp $\mathrm{CH}$ 27, bacteria halófila moderada aislada de las Salinas de Pilluana (San Martín), hidroliza diferentes aceites vegetales, contiene una lipasa con estabilidad térmica hasta $45{ }^{\circ} \mathrm{C}^{6}$. Por ello, el objetivo de esta investigación fue optimizar las condiciones de inmovilización de esta lipasa, entender la significancia y la interacción de los factores que afectan la actividad específica de la enzima inmovilizada para su uso potencial como biocatalizador. 


\section{PARTE EXPERIMENTAL}

\section{Obtención de la lipasa}

Marinobacter sp $\mathrm{CH} 27$ fue cultivado en medio agua de sales (SW) conteniendo cloruro de sodio $5 \%$, suplementado con extracto de levadura $0,5 \%$, así como aceite de oliva al $1 \%$ y Triton X-100 0,1\% como inductor y emulgente, respectivamente. Las condiciones de fermentación y concentración del crudo enzimático fueron las descritas por Fernández-Jerí $\mathrm{y} \mathrm{col}^{6}$.

\section{Inmovilización de lipasa}

Se procedió a una inmovilización por adsorción interfacial usando el soporte octadecilo sepabead (Sepabead EC-OD; Mitsubishi Chemical Corporation, USA) según lo descrito por Guisan $^{7}$ con las siguientes modificaciones: Se pesaron $0,1 \mathrm{~g}$ del soporte octadecilo sepabead y se procedió a lavar 5 veces con agua destilada estéril, se hidrató con buffer fosfato al $\mathrm{pH}$ y a la concentración salina requerida para cada ensayo a $10^{\circ} \mathrm{C}$ durante $24 \mathrm{~h}$. Este soporte ya hidratado fue contactado con $15 \mathrm{mg}$ de proteína contenida en el crudo enzimático y se mezcló suavemente a $10^{\circ} \mathrm{C}$ por el tiempo establecido en cada experimento. Después se centrifugó a $5000 \mathrm{rpm}$ a $10^{\circ} \mathrm{C}$ por $10 \mathrm{~min}$. Finalmente, se separaron la enzima adsorbida al soporte y el sobrenadante, en este último se determinó proteínas residuales.

\section{Factores que influyen en la inmovilización de la lipasa}

Se empleó la Metodología de Superficie Respuesta (MRS) para optimizar las condiciones de máxima actividad específica de la enzima inmovilizada. El diseño estadístico Box-Behnken fue empleado a 3 niveles y 3 factores, para evaluar los parámetros que intervienen en el proceso de inmovilización. El programa Minitab 16 fue empleado obteniendo un total de 12 experimentos y 3 réplicas en el punto central. Las variables y los niveles seleccionados fueron: fuerza iónica (2,5; 5,0 y 10,0 mM de buffer fosfato), tiempo (30,60 y $120 \mathrm{~min})$ y pH $(6,0 ; 7,0$ y 8,0$)$. Para este análisis se emplearon soluciones de buffer fosfato para obtener los $\mathrm{pH}$ correspondientes.

En cada ensayo de inmovilización se determinó el porcentaje de inmovilización, la relación de $\mathrm{mg}$ de proteína/g de soporte, la actividad específica de la lipasa inmovilizada (U/g soporte) y el rendimiento.

\section{Actividad de la lipasa}

En la determinación de la actividad hidrolítica de la lipasa se usó el p-nitrofenil palmitato (p-NPP) (Sigma, USA) siguiendo la metodología propuesta por Gupta y col. ${ }^{8}$, con las siguientes modificaciones: a 1,6 mg de p-NPP se añadieron $1 \mathrm{~mL}$ de isopropanol $0,1 \mathrm{~mL}$; Triton X-100 y $25 \mathrm{mM}$ de buffer fosfato a $\mathrm{pH} 7$ hasta completar $10 \mathrm{~mL}$ de volumen. Para determinar la actividad enzimática se emplearon $1,75 \mathrm{~mL}$ de la solución antes descrita y 0,25 $\mathrm{mL}$ del biocatalizador. La mezcla se incubó a $37{ }^{\circ} \mathrm{C}$ por $20 \mathrm{~min}$, la hidrólisis del p-NPP se evidenció por el incremento del p-nitrofenol a $410 \eta \mathrm{m}$. Una unidad de actividad enzimática se definió como la cantidad de enzima necesaria para liberar $1 \mu \mathrm{mol}$ de p-nitrofenol en un minuto. 


\section{Porcentaje de inmovilización de la lipasa}

La cantidad de enzima inmovilizada se determinó midiendo las concentraciones en la solución enzimática inicial (mg proteína inicial), el sobrenadante y en las soluciones de lavado (mg proteína final) usando el reactivo de Bradford, según la metodología del fabricante (AMRESCO). A partir de estos datos se calcularon el porcentaje de inmovilización y los mg de proteína/g de soporte. La albúmina sérica bovina (BSA) se utilizó como estándar a las concentraciones de $125,250,500,750$ y $1000 \mu \mathrm{g} / \mathrm{mL}$.

\section{RESULTADOS Y DISCUSIÓN}

La inmovilización de la lipasa producida por Marinobacter sp $\mathrm{CH} 27$ fue obtenida a diferentes $\mathrm{pH}$, tiempos y diferentes valores de fuerza iónica (tabla 1).

Tabla 1. Factores que influyen en el proceso de inmovilización de la lipasa extracelular de Marinobacter sp $\mathrm{CH}$ 27, según diseño estadístico experimental Box-

Behnken de superficie respuesta.

\begin{tabular}{|cccclcl|}
\hline $\begin{array}{c}\text { Experimento } \\
\left(\mathbf{N}^{\circ}\right)\end{array}$ & $\mathbf{p H}$ & $\begin{array}{c}\text { Tiempo } \\
(\mathbf{m i n})\end{array}$ & $\begin{array}{c}\text { Fza. Iónica } \\
\mathbf{( m M )}\end{array}$ & $\begin{array}{l}\text { Proteína } \\
\text { inmovilizada } \\
(\mathbf{m g} / \mathbf{g})\end{array}$ & $\begin{array}{c}\text { Inmovilización } \\
(\mathbf{\%})\end{array}$ & $\begin{array}{l}\text { Actividad } \\
\text { lipasa } \\
\text { inmovilizada } \\
\text { (U/g soporte) }\end{array}$ \\
\hline 1 & & & & & & 1,4250 \\
2 & 6 & 30 & 2,5 & 8,25 & 55,00 & 5,5096 \\
3 & 7 & 30 & 2,0 & 14,30 & 95,33 & 10,6601 \\
4 & 8 & 30 & 5,0 & 14,39 & 95,93 & 4,0875 \\
5 & 6 & 60 & 10,0 & 14,44 & 67,50 & 6,8723 \\
6 & 6 & 120 & 5,0 & 14,61 & 96,26 & 4,9117 \\
7 & 7 & 120 & 2,5 & 14,56 & 97,40 & 7,1122 \\
8 & 8 & 60 & 10,0 & 7,54 & 50,27 & 1,0710 \\
9 & 6 & 60 & 2,5 & 14,59 & 97,27 & 2,1207 \\
10 & 7 & 30 & 10,0 & 14,38 & 95,87 & 10,0083 \\
11 & 8 & 120 & 5,0 & 11,29 & 75,27 & 2,1938 \\
12 & 7 & 120 & 10,0 & 14,63 & 97,50 & 3,3638 \\
13 & 7 & 60 & 5,0 & 14,64 & 97,60 & 6,5777 \\
14 & 7 & 60 & 5,0 & 14,64 & 97,60 & 6,5211 \\
15 & 7 & 60 & 5,0 & 14,42 & 96,13 & 5,7581 \\
\hline
\end{tabular}

El mayor porcentaje de inmovilización $(97,6 \%$ ) se obtuvo en los experimentos 13 y 14 a $\mathrm{pH} 7,0 ; 60 \mathrm{~min}$ y fuerza iónica $5 \mathrm{mM}$, y la actividad enzimática máxima después de la inmovilización fue de 10,66 U/g-soporte determinado en el experimento 3 a pH 7,0; $30 \mathrm{~min}$ y fuerza iónica de $2,5 \mathrm{mM}$.

En la figura la se observa el efecto de la fuerza iónica sobre la adsorción de las proteínas al soporte. Así, la máxima adsorción se obtuvo a la concentración de $6 \mathrm{mM}$; en cambio, la actividad máxima de la enzima inmovilizada fue a 2,5 $\mathrm{mM}$ (figura 1d). 
a)

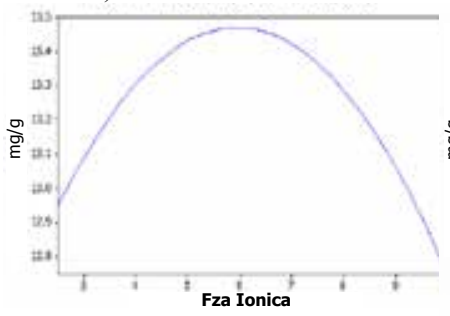

d)

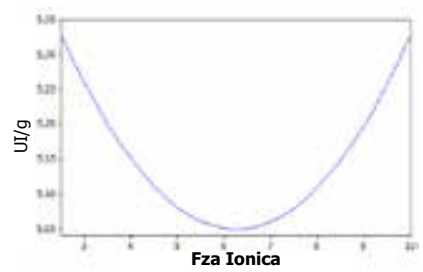

b)

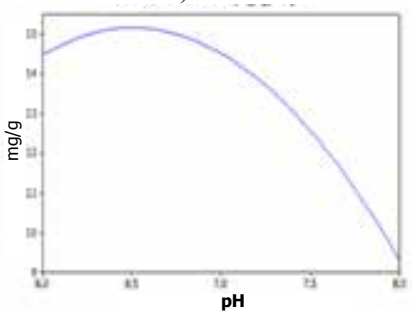

e)

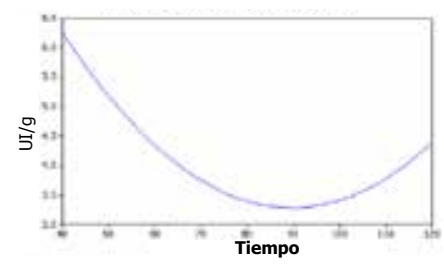

c)

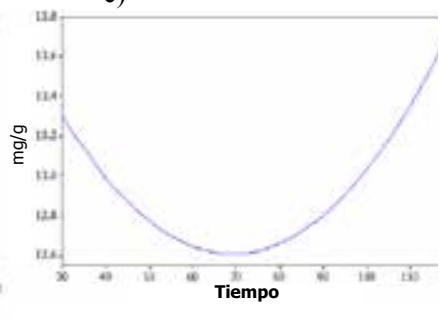

f)

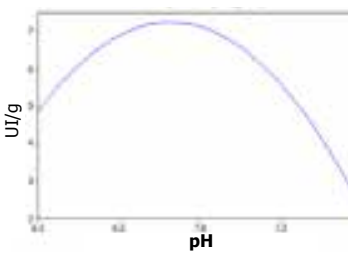

Figura 1. Factores que influyen en la adsorción de la proteína al soporte (a, b y c) y en la actividad específica de la lipasa inmovilizada en octadecilo sepabead durante el proceso de inmovilización (d, e y f)

En las figuras 1c y e se observan el comportamiento de la inmovilización y el tiempo mediante el gráfico de la campana invertida, la adsorción de la enzima al soporte octadecilo sepabead fue antes de los $30 \mathrm{~min}$; pero a medida que el tiempo aumentó la adsorción disminuyó hasta el $84 \%$. Cabe resaltar que a los 70 min de nuevo se incrementó la adsorción de la proteína indicando la presencia de otra lipasa.

El sistema lipasa: octadecilo sepabead fue afectado por el $\mathrm{pH}$, evidenciado en la campana de Gauss generada tanto para la proteína inmovilizada como para la actividad de la lipasa inmovilizada (figuras $1 \mathrm{~b}$ y f). La máxima actividad de la lipasa inmovilizada fue a $\mathrm{pH}$ 7, 0 con $7 \mathrm{U} / \mathrm{g}$ soporte, correspondiendo al $89 \%$ de la enzima adsorbida al soporte.

La fuerza iónica es un parámetro importante para el proceso de inmovilización por la interacción hidrofóbica, de este modo la adsorción de las proteínas se incrementa con la fuerza iónica; sin embargo, las diferentes concentraciones de los electrolitos que proveen la fuerza iónica pueden afectar la actividad enzimática ${ }^{8,9}$. Por lo tanto, este es un parámetro que requiere optimización. Así, a la fuerza iónica mayor de $6 \mathrm{mM}$ disminuyó el rendimiento de inmovilización, este fenómeno puede explicarse por la desorción de la proteína adsorbida por efecto de la alta fuerza iónica, de igual forma Fernández-La Fuente y col. ${ }^{10}$; Bastida y col. ${ }^{11}$; Montero ${ }^{12}$, reportaron disminución de la adsorción de la lipasa cuando se incrementa la fuerza iónica. Además, la desorción depende de la enzima, el soporte, el pH y la temperatura. 
La rápida adsorción de la enzima al soporte sugiere que la proteína cargada se localizó en la superficie externa del soporte, en lugar de ser distribuida uniformemente a través de los poros del mismo. Tiempos de inmovilización cortos han sido reportados por Montero y col., ${ }^{12}$; quienes inmovilizaron el $64 \%$ de lipasa de Candida rugosa en soporte de polipropileno en 1 min.

Las gráficas de contorno muestran la relación entre dos variables independientes a la vez y su efecto en la respuesta de la actividad específica de la lipasa inmovilizada y la proteína inmovilizada en el soporte hidrofóbico octadecilo sepabead (figuras 2 y 3 ).

(a)

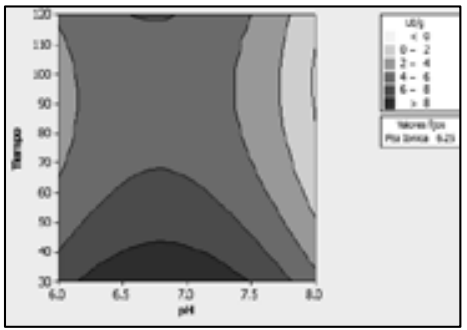

(b)

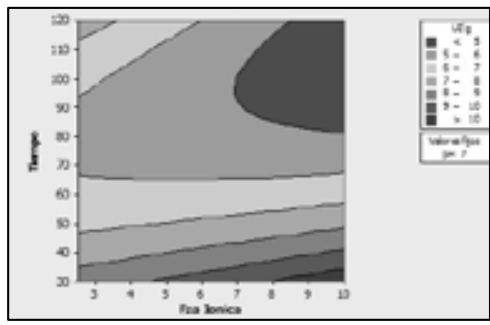

(c)

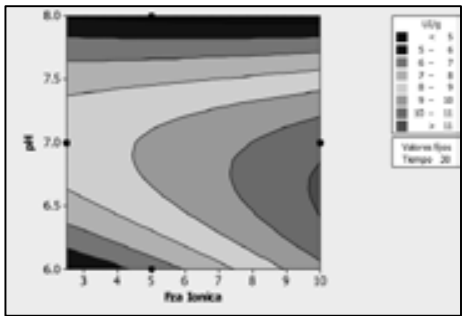

Figura 2. Gráficas de contorno de dos factores, de la actividad específica de la lipasa inmovilizada $(\mathrm{U} / \mathrm{g})$ frente $\mathrm{a}$ : a) tiempo y $\mathrm{pH}, \mathrm{b}$ ) tiempo y fuerza iónica, c) $\mathrm{pH}$ y fuerza iónica. 
(a)

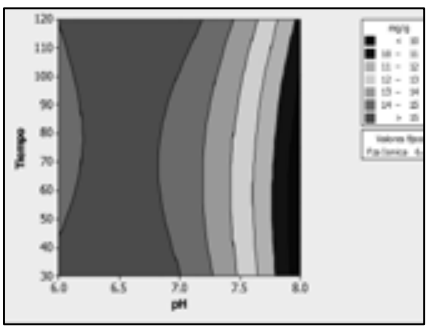

(c)

(b)
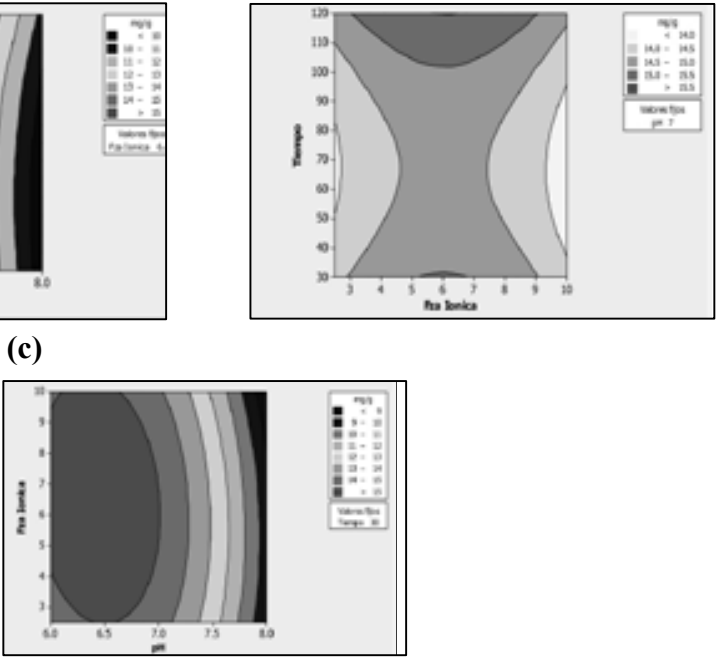

Figura 3. Gráficas de contorno de dos factores, de la proteína inmovilizada $(\mathrm{mg} / \mathrm{g})$ frente a: a) tiempo y $\mathrm{pH}, \mathrm{b}$ ) tiempo y fuerza iónica, c) $\mathrm{pH}$ y fuerza iónica.

Al variar el tiempo y el pH simultáneamente (figuras 2a y 3a), se evidencia que la máxima actividad específica de la lipasa inmovilizada es mayor que $8 \mathrm{U} / \mathrm{g}$, obtenido en el rango de $\mathrm{pH}$ de 6,0 a 7,5 a los $30 \mathrm{~min}$. Por encima de estos valores, la actividad disminuye durante la inmovilización. Estos dos factores son importantes para la actividad de la lipasa durante el proceso de inmovilización. Sin embargo, las mismas variables de tiempo y $\mathrm{pH}$ frente a la proteína inmovilizada no presentan la misma respuesta, si bien la mayor concentración de proteína inmovilizada se presentó en el rango de $\mathrm{pH}$ entre 6,0 a 7,0; el tiempo de mayor respuesta fue sostenido entre 30 a $120 \mathrm{~min}$. Estos resultados se sustentan por el análisis Anova que indica al $\mathrm{pH}$ como la única variable significativa $(\mathrm{P}=0,00)$ en la adsorción de la enzima al soporte, además no existe interacción entre las variables $\mathrm{pH}$ y tiempo $(\mathrm{P}=0,353)$. Con relación al tiempo y fuerza iónica simultáneamente sobre las variables respuestas no se generaron líneas de contorno (figuras $2 \mathrm{~b}$ y $3 \mathrm{~b}$ ).

En las figuras 2c y 3c, se observa que la máxima actividad específica de la lipasa inmovilizada fue mayor de $11 \mathrm{U} / \mathrm{g}$ soporte obtenido a $\mathrm{pH}$ mayor que 6,5 y $10 \mathrm{mM}$ de fuerza iónica. En tanto que la máxima respuesta de proteína inmovilizada fue a $\mathrm{pH}$ 6,5 entre 4 y $10 \mathrm{mM}$. Según, el análisis de varianza no existe interacción entre las variables $\mathrm{pH}$ y fuerza iónica para ambos casos $(\mathrm{P}>0,05)$. Además, la variación de la fuerza iónica en el rango estudiado no influye en el proceso de inmovilización. 
La proteína adsorbida a los soportes depende de varios parámetros tales como el tipo de lipasa, la superficie del soporte, las condiciones ambientales y los protocolos de inmovilización. Nuestros resultados evidencian que no existe relación entre la proteína inmovilizada y la actividad específica de la enzima inmovilizada, ya que al incrementar las concentraciones de proteína adsorbida al soporte, la actividad de la enzima disminuye, esto se explica por la interacción proteína-proteína que inactiva el centro activo de la lipasa. Resultados similares ha descrito Yüce13 en la optimización de la inmovilización de la lipasa de Thermomyces lanuginosuse con relación a las concentraciones de enzima, buffer y $\mathrm{pH}$ frente a la actividad específica.

El diseño estadístico experimental Box-Behnken de superficie respuesta fue empleado para optimizar los parámetros de inmovilización de la lipasa producida por Marinobacter sp CH27. La optimización se realizó para la actividad específica de la lipasa inmovilizada. El ANOVA se presenta en la tabla 2 , el modelo se ajusta a un polinomial de segundo orden $(\mathrm{P}=$ $0,020)$. Representa la actividad de hidrólisis de $\mathrm{pNPP}$ (U/g soporte) como una función del $\mathrm{pH}$ (X1), tiempo (X2), fuerza iónica (X3) y es expresado por la siguiente ecuación:

$\mathbf{Y}=51.2763 \mathbf{X}_{1}-0.1078171 \mathbf{X}_{2}+2.88701 \mathbf{X}_{3}-3.58600 \mathbf{X}_{1}{ }^{2}+0.00107060 \mathbf{X}_{2}{ }^{2}+0.00774500$ $\mathbf{X}_{3}{ }^{2}-0.00631556 \mathbf{X}_{1} \mathbf{X}_{2}-0.3521247 \mathbf{X}_{1} \mathbf{X}_{3}-0.00778456 \mathbf{X}_{2} \mathbf{X}_{3}$

Tabla 2. Análisis de varianza de la actividad específica de la lipasa inmovilizada en octadecilo sepabead.

\begin{tabular}{|lccccc|}
\hline Fuente & GL & SC Sec. & MC & F & P \\
& \multicolumn{5}{c|}{ Ajust. } \\
\hline Regresión & 9 & 105,558 & 11,7287 & 4,75 & \\
& & & & & $0,050^{* *}$ \\
Lineal & 3 & 27,599 & 14,4299 & 5,84 & 0,043 \\
pH & 1 & 14,147 & 18,3562 & 7,43 & \\
tiempo (min) & 1 & 13,444 & 24,7956 & 10,03 & $0,042^{* *}$ \\
Fza. iónica & 1 & 0,008 & 0,3698 & 0,15 & \\
Cuadrática & 3 & 62,790 & 21,5548 & 8,72 & $0,025^{* *}$ \\
pH*pH & 1 & 49,740 & 47,3594 & 19,16 & 0,715 \\
tiempo *tiempo & 1 & 13,017 & 13,0431 & 5,28 & \\
Fza. iónica*Fza. I & 1 & 0,033 & 0,0329 & 0,01 & $0,020^{* *}$ \\
Interacción & 3 & 15,170 & 5,0565 & 2,05 & $0,007^{* *}$ \\
pH* tiempo & 1 & 0,195 & 0,3401 & 0,14 & 0,070 \\
pH* Fza. iónica & 1 & 7,305 & 7,3051 & 2,96 & 0,091 \\
tiempo*Fza. iónica & 1 & 7,670 & 7,6696 & 3,10 & 0,226 \\
Error residual & 5 & 12,358 & 2,471 & & 0,726 \\
& & & & & 0,146 \\
& & & & & 0.138 \\
& & & & & \\
& &
\end{tabular}

** Estadísticamente significativo 
Finalmente, se optimizaron los parámetros y se alcanzó la actividad máxima de 11,16 U/g soporte durante la inmovilización de lipasa en octadecilo sepabead a $\mathrm{pH}$ de 6,62; 30 min y fuerza iónica de $10 \mathrm{mM}$, con una actividad relativa obtenida de $81,50 \%$, y 74,0\% de rendimiento de inmovilización de lipasa en ocatadecilo sepabead.

Las técnicas de adsorción, han demostrado producir biocatalizadores que presentan altas retenciones de actividades catalíticas, aunque este principio es válido sólo para enzimas puras. La inmovilización de extractos heterogéneos, por ejemplo, mezclas de proteínas o extractos crudos, usando métodos de adsorción interfacial en soportes hidrofóbicos, han demostrado ser procesos selectivos de inmovilización de lipasas ${ }^{10}$. Además, esta técnica permite en un solo paso inmovilizar, concentrar, purificar e hiperactivar concentraciones pequeñas de lipasas presentes en extractos $\operatorname{crudos}^{4,10,14,15}$. En este aspecto, Palomo y col.15 emplearon la combinación de dos soportes hidrofóbicos: octil-agarosa y octadecilo sepabead con los que lograron purificar en octil-agarosa e inmovilizar en octadecilo sepabead la lipasa de Bacillus thermocatenulatus (BTL2).

El soporte hidrofóbico octadecilo sepabead se parece a la interface formada por los sustratos naturales de la lipasa. La adsorción interfacial puede producir un congelamiento de la estructura de la enzima en su forma abierta y de esta manera incrementar la aparente afinidad de la enzima por el sustrato ${ }^{14}$, además de la hiperactivación. Los resultados obtenidos demuestran una adsorción selectiva al soporte hidrofóbico pero no se produce hiperactivación marcada como describen diversos autores ${ }^{11,14,15}$. En este estudio se usó para-nitrofenil palmitato como sustrato, un ácido carboxílico de cadena larga; por el contrario, Palomo y col. ${ }^{14}$ emplearon la adsorción hidrofóbica para inmovilizar las lipasas de Candida antartica (fracción B), Mucor miehei y Candida rugosa a octadecilo-sepabead y usaron como sustratos para-nitrofenil propionato y etil-butirato, de esta forma demostraron hiperactivación de 2 a 5 veces, para etil-butirato pero no con él para-nitrofenil propionato, probablemente el etil butirato, al ser un compuesto más pequeño, puede acceder fácilmente al centro activo, por lo tanto la hiperactivación depende de la fuente de enzima y del sustrato.

\section{CONCLUSIONES}

Se optimizo el proceso de inmovilización de la lipasa producida por Marinobacter sp $\mathrm{CH} 27$ a las condiciones de $\mathrm{pH}$ 6,62; 30 min y fuerza iónica de $10 \mathrm{mM}$, con una actividad relativa de $81,50 \%$, y rendimiento de inmovilización de lipasa en ocatadecilo sepabead de $74,0 \%$

\section{AGRADECIMIENTO}

Este trabajo fue financiado por la subvención $\mathrm{N}^{\circ}$ 031-2011- CONCYTEC - OAJ y contrato No 017 -FINCYT - PIPAB - 2008 


\section{REFERENCIAS BIBLIOGRÁFICAS}

1. Jaeger K-E, Eggert T. Lipases for biotechnology. Curr Opin Biotechnol 2002; 13:390 397

2. Pandey A, Benjamin S, Soccol CR, Nigam P, Krieger N, Soccol UT The realm of microbial lipases in biotechnology. Biotechnol Appl Biochem 1999; 29:119-131

3. González-Bacerio, J; Rodríguez Hernández, J; Monte Martínez, A. Las lipasas: enzimas con potencial para el desarrollo de biocatalizadores inmovilizados por adsorción interfacial. Rev Colomb Biotecnol 2010; 12(1): 124-40

4. Bastida, A., Sabuquillo, P., Armisen, P., Fernández-Lafuente, R., Huguet, J., Guisán, J. M. A single step purification, immobilization and hyperactivation of lipases via interfacial adsorption on strongly hydrophobic supports. Biotechnol Bioeng 1998; 58 (5): 486-93

5. Resindion S.R.L. Mitsubishi Chemical Corporation, [homepage on the Internet]. Tokio: Resindion S.R.L; c2015 [updated 2015 Sep; cited 2016 Nov 16]. Available from: http:// www.resindion.com/images/stories/resindion/download/RESINDION_brochure_ ReliZyme_SepabeadsEC_set2015_web.pdf

6. Fernández-Jerí, Y; Zavaleta, A.I; Alejandro-Paredes, Luis; Izaguirre, Víctor. Caracterización parcial de una lipasa extracelular de Marinobacter sp. Empleando la Metodología de Superficie Respuesta. Cienc Invest 2013; 16 (1): 12-17

7. Brena, B.M. y F. Batista-Viera, Methods in Biotechnology: Immobilization of Enzymes and Cells, 2nd Ed. Edited by: J. M. Guisan (C Humana Press Inc. Totowa: NJ; 2006.

8. Gupta R, Rathi P, Gupta N, Bradoo S. Lipase assays for conventional and molecular screening: an overview. Biotechnol Appl Biochem 2003; 37 (1):63-71

9. Oztürk B. Immobilization of Lipase from Candida rugosa on Hydrophobic and Hydrophilic Supports. [Doctoral dissertetion]. Izmir: Izmir Institute of Technology, Department of Biotecnology and Bioengenery 2001

10. Fernández-Lafuente R, Armisén P, Sabuquillo P, Fernández-Lorente P, Guisan J.M. Immobilization of lipases by selective adsorptionon hydrophobic supports. Chem Phys Lipids 1998; 93 (1): 185-197

11. Bastida, A., Sabuquillo, P., Armisen, P., Fernández-Lafuente, R., Huguet, J., Guisán, J. M. A single step purification, immobilization and hyperactivation of lipases via interfacial adsorption on strongly hydrophobic supports. Biotechnol Bioeng 1998; 58 (5): 486-93.

12. Montero S, Blanco A, Virto M, Ladenta L C, Agud I, Solozabal R, et al. Immobilization of Candida rugosa lipase and some properties of the immobilized enzyme., Enzyme Microb Technol 1993; 15(3): 239-247

13. Yücel Y. Optimization of immobilization conditions of Thermomyces lanuginosus lipase on olive pomace powder using response surface methodology. Biocatalysis and Agric Biotechnol 2012; 1(1) :39-44.

14. Palomo J. M, Muñoz G, Fernández-Lorente G, Mateo C, Fernández-Lafuente R, and Guisán J. M. Interfacial adsorption of lipases on very hydrophobic support (octadecylSepabeads): immobilization, hyperactivation and stabilization of the open form of lipases. J Mol Catal B Enzyme 2002; 19: 279-286 
15. Palomo J. M, Segura R. L, Fernández-Lorente G. Pernas M, Rua M. L, Guisán J. M, et al, Immobilization, and Stabilization of a Lipase from Bacillus thermocatenulatusby Interfacial Adsorption on Hydrophobic Supports. Biotechnol Prog 2004; 20 (2):630-635 